\title{
A MULTIOBJECTIVE MODEL FOR PASSIVE PORTFOLIO MANAGEMENT: AN APPLICATION ON THE S\&P 100 INDEX
}

\author{
Fernando García ${ }^{1}$, Francisco Guijarro ${ }^{2}$, Ismael Moya ${ }^{3}$ \\ Departmento de Economía y Ciencias Sociales, Universidad Politécnica de Valencia, \\ Cami de Vera s/n, 46022 Valencia, Spain \\ E-mails: '1fergarga@esp.upv.es (corresponding author); 2 fraguima@upvnet.upv.es; \\ 3imoya@esp.upv.es
}

Received 01 December 2011; accepted 20 February 2012

\begin{abstract}
Index tracking seeks to minimize the unsystematic risk component by imitating the movements of a reference index. Partial index tracking only considers a subset of the stocks in the index, enabling a substantial cost reduction in comparison with full tracking. Nevertheless, when heterogeneous investment profiles are to be satisfied, traditional index tracking techniques may need different stocks to build the different portfolios. The aim of this paper is to propose a methodology that enables a fund's manager to satisfy different clients' investment profiles but using in all cases the same subset of stocks, and considering not only one particular criterion but a compromise between several criteria. For this purpose we use a mathematical programming model that considers the tracking error variance, the excess return and the variance of the portfolio plus the curvature of the tracking frontier. The curvature is not defined for a particular portfolio, but for all the portfolios in the tracking frontier. This way funds' managers can offer their clients a wide range of risk-return combinations just picking the appropriate portfolio in the frontier, all of these portfolios sharing the same shares but with different weights. An example of our proposal is applied on the S\&P 100 .
\end{abstract}

Keywords: index tracking, frontier curvature, tracking error variance, excess return, portfolio variance, mean-variance model, portfolio selection.

Reference to this paper should be made as follows: García, F.; Guijarro, F.; Moya, I. 2013. A multiobjective model for passive portfolio management: an application on the S\&P 100 index, Journal of Business Economics and Management 14(4): 758-775.

JEL Classification: C61; G11; G14.

\section{Introduction}

The increasing popularity of passive portfolio techniques is probably due to the difficulty to model and predict the evolution of stock markets (Jarrett, Schilling 2008; Teresiene 2009; Aktan et al. 2010). Index tracking seeks to minimize the unsystematic risk component by imitating the movements of a reference benchmark - a stock index. Faced with active management techniques that endeavor to beat the underlying index, tracking portfolios in general and tracking indices in particular, are configured as a powerful passive strategy. 
Index Tracking can be full or partial depending on the number of stocks that are considered.

In the case of full tracking, the portfolio includes the same stocks as the index, and an exact tracking is produced if these stocks are weighted in the same proportion as the index. The disadvantages of full tracking include the high portfolio management and transaction costs, as well as the need to invest in all the stocks in the index despite they might have a minor weight in the index composition. Various other drawbacks are mentioned in the literature (Ruiz-Torrubiano, Suárez 2009). A restrictive view of the costs associated with tracking portfolios has also been discussed in numerous academic papers (Connor, Leland 1995; Canakgoz, Beasley 2003) and the drawbacks are usually addressed through mathematical programming models.

In partial tracking a manager builds a portfolio from a subset of stocks contained in the underlying index and this process removes some of the drawbacks listed above.

Three issues must be resolved when building a partial tracking.

Firstly, the number of stocks in the tracking must be chosen. An evaluation can be made using sensitivity analysis on the results to contrast the desirability of increasing or decreasing the cardinality of the tracking portfolio (Tabata, Takeda 1995).

After setting the number of stocks, the second question involves selecting the stocks among the available ones. The simplest approach is to assess each potential stock, to measure the index tracking error, and then select those stocks that minimize this deviation. Unfortunately this approach is computationally difficult because it represents an NP-hard problem (Ruiz-Torrubiano, Suárez 2009).

Finally, the third question involves the precise weight to be given to each stock in the tracking portfolio, depending on the desired return and the tracking error the manager is willing to assume.

The second issue of stock selection has received special attention from researchers and many methods for finding the local problem optimum have been proposed. These methods can be grouped into two broad families: those that make use of mathematical programming, and those using multivariate analysis techniques.

Without being exhaustive, authors using mathematical programming models for optimal local searches include: Tabata and Takeda (1995), whose approach is employed in this paper and discussed in a later section; Beasley et al. (2003), whose approach uses a population heuristic in which the cardinality of the portfolio is made explicit through the restriction $\sum_{i=1}^{N} z_{i}=n, n$ being the number of stocks in the tracking portfolio, and $z_{i}$ a binary variable that indicates if the $i$-th stock is to be included in the portfolio or not; Derigs and Nickel (2004) use a procedure of Simulated Annealing; Ruiz-Torrubiano and Suárez (2009) combine a genetic algorithm with a model of quadratic programming in a more general formulation of the problem; Gaivoronoski et al. (2005) use different measures of risk in mathematical programming models, such as return variance, semivariance, tracking error variance, or value at risk (VAR). 
Works that make use of multivariate analysis techniques include: Focardi and Fabozzi (2004), Dose and Cincotti (2005), Corielli and Marcellino (2006).

All these papers are characterized by the search for a single portfolio, characterized up to three possible parameters: tracking error variance, excess return and volatility of returns, which represent reliability, profitability and risk (Rutkauskas, Stasytyte 2007). The stocks in the tracking portfolio are identified during this process and the given weighting complies with the constraints imposed on those parameters.

This paper proposes the addition of a new parameter: the curvature of the mean-variance frontier. This criterion is not defined for a given portfolio, but for the set of portfolios that define the tracking frontier. The main advantage is that a fund manager can satisfy different investment profiles using the same subset of stocks - with all the portfolios on the frontier containing the same stocks and so reducing transaction costs - and can also simultaneously consider different criteria in the tracking index problem.

Usually partial tracking portfolio models have attempted to obtain a single portfolio that will only satisfy those investors whose profile is perfectly aligned with the configuration chosen by the portfolio manager. If the investment profile changes, then the portfolio also changes the stocks employed and not only the weights.

The rest of the paper is structured as follows. The second section analytically presents the three key concepts for tracking indices: tracking error variance, excess return, and portfolio variance. The following section introduces a new criterion, the curvature of the tracking frontier, and discusses the benefits that arise from adding the concept of gradient to the previous ones. The fourth section presents a multiobjective programming model for generating tracking frontiers by simultaneously considering all these parameters. In addition, various other propositions regarding the curvature of the tracking frontier are discussed and demonstrated. In the fifth section, the above model is applied to the partial tracking of the S\&P 100. A summary of the main conclusions is presented in the final section.

\section{Parameters in the tracking portfolio problem: tracking error variance, excess return, and portfolio variance}

Tracking error is defined as the absolute difference between tracking portfolio returns and the returns produced by the tracked index. Since the aim is for both portfolios to maintain a parallel evolution over time, the problem is posed as a minimization of the volatility in the tracking error. A reduction in the volatility of the tracking error means minimizing the variance in returns between the tracking portfolio and the stock index (Roll 1992). In this way, a clear parallel with the mean-variance model (Markowitz 1952, 1959) is established. However, with the difference that instead of looking for the portfolio with the least volatility for a given return, managers try to obtain the portfolio with the minimum tracking error variance for a given level of return in excess of the index. These are the foundations of the TEV (Tracking Error Variance) criterion: 
(1) minimize the TEV; (2) assume a certain TE (Tracking Error ${ }^{1}$ ). Both objectives are inherently conflicting, so the manager should look for compromise solutions.

The TEV is given by the expression (1):

$$
T E V=\mathbf{x}^{t} \mathbf{V} \mathbf{x},
$$

where: $\mathbf{x}$ - vector of dimension $N \times 1$, contains the weightings difference of the $N$ stocks between the tracking portfolio and the index; that is, $\mathrm{x}=\mathrm{x}_{p}-\mathrm{x}_{b}$, where $\mathrm{x}_{p}$ is the vector of weightings in the tracking portfolio and $x_{b}$ is the weighting vector in the index (subscript $b$ for benchmark). A full tracking is obtained if all elements of $\mathrm{x}$ are zero, while non-zero deviations can take risk-return positions that differ from the index. In the partial tracking, the vector $\mathrm{x}_{p}$ will have the same number of non-zero elements as there are stocks included in the tracking, $n$ and the remaining weights will be left with a value of zero. $\mathbf{V}$ - variance-covariance matrix for the stocks returns.

The excess return $G$ on the index is obtained as the difference between the returns of the tracking portfolio and the index (2):

$$
G=x^{t} R=x_{p}^{t} R-x_{b}^{t} R=R_{p}-R_{b}
$$

where: $R$ - vector of returns of $\mathrm{N}$ stocks. $R_{p}\left(R_{b}\right)$ - returns of the tracking portfolio (index).

Unlike other models, in the tracking portfolio the return in excess $G$ is obtained by subtracting the index return, and not the return of the risk-free asset. The full tracking can be easily resolved by using a quadratic mathematical model (3):

$$
\begin{aligned}
\text { Min }= & x^{t} V x, \\
\text { s.t. } & x^{t} R=G, \\
& x^{t} 1=0,
\end{aligned}
$$

where: 1 - vector of dimension $N \times 1$ with all the elements 1 .

Note the need to explicitly include the constraint on $G$, since the profitability of the tracking portfolio and the index can differ by a constant, and the value of the TEV could paradoxically be zero. The second constraint ensures that the total investment in the tracking portfolio is the same as the index - and so the sum of positive and negative deviations is compensated.

\section{An additional parameter: the curvature of the TEV frontier}

Model (3) enables to obtain different portfolios depending of the value of excess return $G$. These different portfolios are obtained by varying the weights of the stocks, and/or

\footnotetext{
${ }^{1}$ Alternatively, Rudolf et al. (1999) suggest using linear measurements of tracking error, and propose the use of goal programming for solving optimization models. This technique has also been recently used by Wu et al. (2007).
} 
varying the stocks when the tracking is partial. Markowitz's minimum variance frontier and TEV frontier appear in Fig. 1. For the case of the full tracking, Roll (1992) shows that the distance in the axis of the returns variance between the two frontiers is constant $k$ for any value of return $R_{p}$. Therefore, the TEV frontier is a simple shift of Markowitz's frontier in the variance axis, and the inefficiency of the index $b$ can be quantified as $k=\sigma_{b^{*}}^{2}-\sigma_{b}^{2}$ being constant for any portfolio on the tracking frontier.

The above property is not satisfied in the case of partial tracking. Fig. 1 shows two TEV frontiers, each generated by removing a single stock from the tracking. The TEV frontier $\mathrm{TEV}_{-i}\left(\mathrm{TEV}_{-j}\right)$ results from the exclusion of the tracking of the $i$-th stock ( $j$-th). Generally, the removal of one or more stocks from the tracking means a greater TEV without necessarily reducing the efficiency of the portfolios. In the example in the figure, the $\mathrm{TEV}_{-i}$ frontier and the $\mathrm{TEV}_{-j}$ frontier partially improve the efficiency of the original TEV in the mean-variance sense. Specifically, both frontiers generate better risk-returns in portfolios nearer to the $R_{b}$ index than the TEV frontier in the full tracking. If the $\mathrm{TEV}_{-i}$ and $\mathrm{TEV}_{-j}$ frontiers are compared then different results will again be reached according to the considered return. However, it must always be remembered that Fig. 1 only reflects risk and return, and not TEV.

Fig. 1 shows the different curvature of the $\mathrm{TEV}_{-i}$ and $\mathrm{TEV}_{-j}$ frontiers. It is precisely this characteristic that can be very useful for the fund manager. The $\mathrm{TEV}_{-j}$ frontier provides a better risk-return combination than the $\mathrm{TEV}_{-i}$ frontier for portfolios with

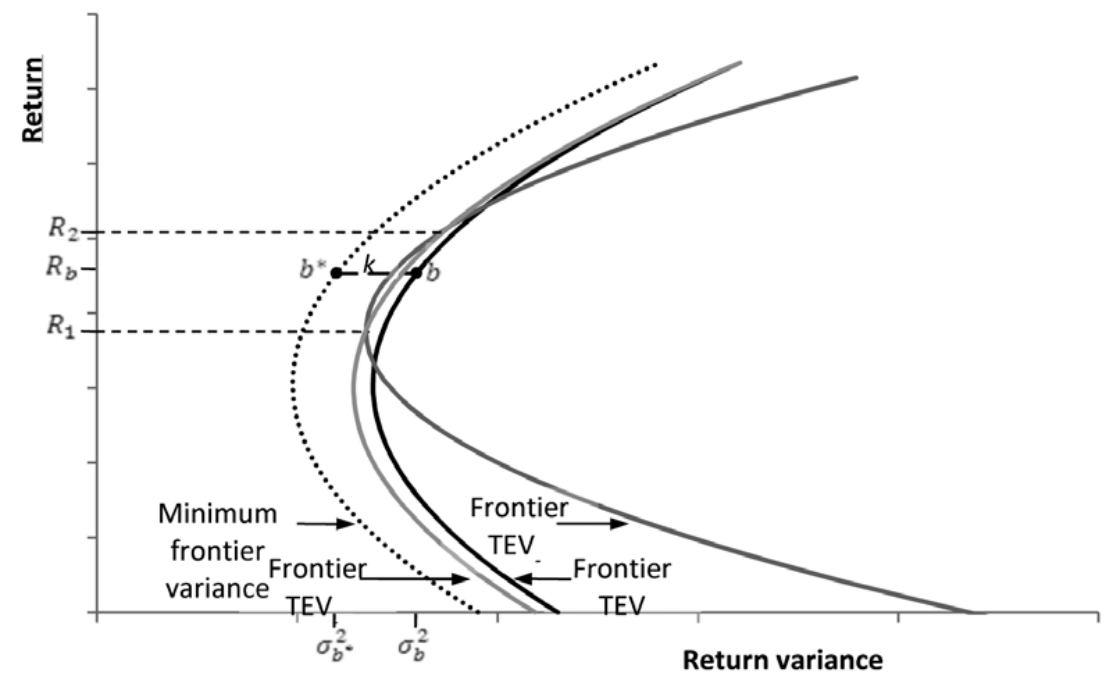

Fig. 1. The minimum variance frontier and various TEV frontiers

Key: …. Minimum variance frontier; — TEV frontier; — TEV frontier excluding the $j$-th stock; - TEV frontier excluding the $i$-th stock; $b$ : position of the index in the mean-variance plane; $b^{*}$ : projection of the index on the minimum variance frontier; $R_{1}$ : return of portfolio 1 (see Roll 1992); $R_{2}$ : return of portfolio 2 (see Roll 1992); $R_{\mathrm{b}}$ : index return; $\sigma_{b}^{2}$ index return variance; $\sigma_{b^{*}}^{2}$ return variance of portfolio $b^{*}$. 
a return of $R_{p} \in\left[R_{1}, R_{2}\right]$ However, for returns outside this range, the $\mathrm{TEV}_{-i}$ frontier generates returns that are clearly better than the portfolios on the $\mathrm{TEV}_{-j}$ frontier. In this situation, the manager must consider which of the two frontiers can best satisfy client profiles. For conservative profiles that intend to simply mimic the index, the $\mathrm{TEV}_{-j}$ frontier is the most suitable, and so the $j$-th stock is removed from the tracking. But if a return in excess $\mathrm{G}$ is required, then the $\mathrm{TEV}_{-i}$ frontier would be the best option. Therefore, not considering the curvature of the tracking portfolio frontier means that the proposed portfolios only satisfy specific values of risk, return and TEV, without considering the possibly varying risk profiles of the fund's clients. When choosing between two tracking frontiers for a given value of $G$ and with the same levels of riskreturn and TEV, the manager must select the frontier with less curvature - because this enables more efficient options to be offered to investors. Examining the curvature of the tracking portfolio enables the manager to make a more global analysis of the offer presented to his/her clients. To achieve this, we propose the entire TEV frontier to be necessarily examined and not just a specific point on it.

We can conclude that the manager will have the following preferences when evaluating tracking portfolios for the criteria presented:

Assumption 1: Investment fund manager preferences:

a. Criteria concerning the tracking portfolio

a. 1 Return: portfolios with higher returns are preferred, ceteris paribus.

a. 2 Returns variance: portfolios with less risk are preferred, ceteris paribus.

a. 3 TEV: portfolios with less TEV are preferred, ceteris paribus.

b. Criteria concerning the TEV frontier

b.1 Curvature of the TEV frontier: TEV frontiers with less curvature are preferred, ceteris paribus.

The following section presents a multiobjective mathematical programming model that enables the simultaneous consideration of all these preferences. This methodology has been widely published in the field of operations research (Zeleny 1982; Steuer 1986), and is currently used in many financial applications (Hallerbach, Spronk 2002).

\section{A multiobjective approach to the problem of partially tracking portfolios}

It is possible to consider the TEV frontier curvature, along with other criteria already referred to in the literature (excess return, return variance, and TEV) into the utility function (4):

$$
U(p)=w_{0} R_{p}-w_{1} \sigma_{p}^{2}-w_{2} \mathrm{TEV}_{p}-w_{3} \kappa_{f}
$$

where: $\kappa_{f}$ - represents the curvature of the TEV frontier, of which portfolio $p$ forms a part; $w_{i}$ - weights of each criteria, with $i=0 \ldots 3$.

Note that the curvature is defined on a frontier $f$, and not on a given portfolio $p$, since the curvature is the same for all portfolios on the frontier (the returns variance and the TEV are quadratic functions). 
Given that in the tracking portfolios the manager fixes a value for the parameter $G$, all of the portfolios evaluated with utility function (4) obtain the same return $R_{p}=R_{b}+G$. In this way, (4) can be simplified as (5):

$$
U(p)=w_{1} \sigma_{p}^{2}-w_{2} \mathrm{TEV}_{p}-w_{3} \kappa_{f}
$$

For convenience, the proposed model will be presented as a minimization problem (6):

$$
\operatorname{Max} U(p) \equiv \operatorname{Min}(-U(\mathrm{p})) \equiv \operatorname{Min} w_{1} \sigma_{p}^{2}+w_{2} \mathrm{TEV}_{p}+w_{3} \kappa_{f}
$$

The multiobjective mathematical programming model is (7):

$$
\begin{aligned}
\text { Min } & w_{1} x_{p}^{t} V x_{p}+w_{2} x^{t} V x+w_{3} \kappa_{f}, \\
\text { s.t. } & x^{t} R=G, \\
& x^{t} 1=0, \\
& x_{p}=x_{b}+x,
\end{aligned}
$$

where the only unknown element is the weightings vector $x$. Note that no restrictions are included on the cardinality of the tracking portfolio. For the application of model (7) it is necessary to address three issues. The first relates to how to find a good solution within the exponential number of portfolios that can be formed and limiting to $n$ the number of stocks in the tracking portfolio. The objective of model (7) is to make a comparison between these portfolios using the utility function, and not to generate a frontier. The second question to address is how to calculate $\kappa_{f}$, the only parameter that has not yet been derived analytically. Finally, there remains the determination of the $w_{i}$ weights in the utility function. Each of these questions is discussed separately in the following subsections.

\subsection{Search for local optima}

The greatest computational burden when solving an instance of model (7) is calculating the curvature of the TEV frontier, as shown in the following paragraph. In the example developed in a later section for the tracking of the S\&P 100 an adaptation of the algorithm proposed by Tabata and Takeda (1995) has been used. This algorithm was chosen because it is simple to implement and generates good local optima. The algorithm ensures that the solution found cannot be improved unless two or more stocks are changed in the tracking portfolio. For a better understanding of the overall process, we present the adaptation of the algorithm ${ }^{2}$ to the multiobjective mathematical programming model (7) (Algorithm 1).

Algorithm 1. Adaptation of the algorithm by Tabata and Takeda (1995)

Definitions:

$V A R_{p}(j, i)$ - change in return variance in tracking portfolio $p$ after substituting the $\mathrm{i}$-th stock for the $j$-th stock. 2 The adaptation of the Tabata and Takeda (1995) algorithm has been programmed in R version 2.2.0.
The authors will provide the code on request. 
$T E V_{x}(j, i)$ - change in the TEV after substituting $i$-th stock for the $j$-th stock in the portfolio with $\mathrm{x}$ weighting vector differences.

$\kappa_{f}(j, i)$ - change in the curvature of the TEV frontier after substituting the $i$-th stock for the $j$-th stock.

$\mathrm{F}(j, i)$ - function that evaluates the change in the objective function after substituting the $\mathrm{i}$-th stock for the $\mathrm{j}$-th stock in the tracking portfolio. Its value is calculated as $\mathrm{F}(j, i)=w_{1} V A R_{p}(j, i)+w_{2} T E V_{\mathbf{x}}(j, i)+w_{3} \kappa_{f}(j, i)$.

$S^{(m)}(n)=$ set of stocks included in the tracking portfolio in the $m$-th iteration, where $\mathrm{n}$ represents the cardinality of the portfolio.

Pseudocode:

Step 0. s: $=0$. Let $S^{(s)}(n)$ be the initial set of stocks, $n$ cardinality.

Step 1. If an optimal solution has not been found for the $\mathbf{x}_{n}^{*}$ weighting vector difference of $S^{(s)}(n)$ and for the objective function $F_{\mathbf{x}}^{*}$ it can be obtained using model (7) by considering only those stocks in the $S^{(s)}(n)$ set. Set $j:=n+1$.

Step 2. $\mathbf{x}_{n}^{*}:=\mathbf{x}_{n}^{*(s)}$. For $S_{j, i}^{\prime(s)}(n),(i=1 \ldots n)$ calculate $F_{\mathbf{x}}^{*}-F_{\mathbf{x}}(j, i)$.

If $F_{\mathbf{x}}^{*}-F_{\mathbf{x}}(j, q)=\max \left\{F_{\mathbf{x}}^{*}-F_{\mathbf{x}}(j, i)\right\}>0$, go to step 3. Otherwise, $j:=j+1$. If $j>N$ then $j:=n+1, i:=1$, go to step 4 .

Step 3. $s:=s+1, S^{(s)}(n):=S_{j, q}^{\prime(s-1)}(n)$. Return to step 1 .

Step 4. For $S_{j, i}^{\prime(s)}(n)$ calculate $\mathbf{x}_{n}^{\prime *(s)}$ and its corresponding $F_{\mathbf{x}^{\prime}}(j, i)$. If $F_{\mathbf{x}}^{*}-F_{\mathbf{x}^{\prime}}(j, i)>0$, then set $s:=s+1, S^{(s)}(n):=S_{j, i}^{(s-1)}(n)$ and return to step 1. Otherwise, perform $i:=i+1$. If $i \leq n$, then $j:=j+1$. If $j \leq n$, set $i:=1$ and repeat step 4. If $j>n$, the current solution $S^{(s)}(n)$ and $\mathbf{x}_{n}^{*}$ is the optimal local solution for building a tracking portfolio with n stocks: STOP.

\subsection{The TEV frontier curvature}

As Roll (1992) demonstrated, the full tracking TEV frontier is a shift of Markowitz's minimum variance frontier, and the curvatures of both frontiers necessarily coincide (Fig. 1). This section sets out various propositions, including one that shows that the curvature of the TEV frontier generated from a subset of $n$ stocks matches the curvature of the minimum variance frontier generated from the same $n$ stocks.

The variance of a minimum variance portfolio $p$ can be obtained by analytically solving Markowitz's mean-variance model (8).

$$
\begin{aligned}
\operatorname{Min}= & \frac{1}{2} \mathrm{x}_{p}^{t} V x_{p}, \\
\text { s.a. } & \mathrm{x}_{p}^{t} R=R_{p}, \\
& \mathrm{x}_{p}^{t} 1=1 .
\end{aligned}
$$

Using the Lagrangian (9) method on this model, we can derive expression (10). 


$$
\begin{gathered}
\mathcal{L}=\frac{1}{2} x_{p}^{t} V x_{p}+\lambda_{1}\left(x_{p}^{t} R-R_{p}\right)+\lambda_{2}\left(x_{p}^{t} 1-1\right), \\
\mathrm{x}_{\mathrm{p}}=V^{-1}\left[\begin{array}{ll}
R & 1
\end{array}\right] \mathrm{A}^{-1}\left[\begin{array}{c}
R_{p} \\
1
\end{array}\right],
\end{gathered}
$$

where $A=\left[\begin{array}{ll}R & 1\end{array}\right]^{t} V^{-1}\left[\begin{array}{ll}R & 1\end{array}\right]=\left[\begin{array}{ll}a & b \\ b & c\end{array}\right], a=R^{t} V^{-1} R, b=R^{t} V^{-1} 1$ and $c=1^{t} V^{-1} 1$. We can express the variance of the $p$ portfolio using (10) such as $\sigma_{p}^{2}=\mathbf{x}_{p}^{t} V \mathbf{x}_{p}$, and developing its expression to arrive at a result which depends on $a, b$ and $c(11)$ :

$$
\begin{aligned}
& \sigma_{p}^{2}=\mathbf{x}_{p}^{t} V \mathbf{x}_{p}=\left[\begin{array}{ll}
R_{p} & 1
\end{array}\right] A^{-1}\left[\begin{array}{c}
R \\
1
\end{array}\right] V^{-1} V V^{-1}\left[\begin{array}{ll}
R & 1
\end{array}\right] A^{-1}\left[\begin{array}{c}
R_{p} \\
1
\end{array}\right]= \\
& {\left[\begin{array}{ll}
R_{p} & 1
\end{array}\right] A^{-1}\left[\begin{array}{c}
R_{p} \\
1
\end{array}\right]=\frac{a-2 b R_{p}+c R_{p}^{2}}{a c-b^{2}} .}
\end{aligned}
$$

The $\kappa_{f}$ curvature of the frontier of minimum variance is obtained as the second derivative of $\sigma_{p}^{2}$ ith respect to $R_{p}(12)$ :

$$
\kappa_{f}=\frac{\partial^{2} \sigma_{p}^{2}}{\partial R_{p}}=\frac{2 c}{a c-b^{2}} .
$$

This curvature matches the curvature of the TEV frontier if the tracking is full. If the tracking is partial, the curvature cannot be calculated using the expression (12), as the values of $a, b$, and $c$ are linked to the full set of stocks. Nevertheless, the following proposition shows how the computation is equivalent to the curvature of the minimum variance frontier generated using the same subset of stocks.

Proposition 1. The curvature of the TEV frontier generated from a subset of $n$ stocks has the same curvature as the minimum variance frontier generated from the same subset of stocks ${ }^{3}$.

Proposition 1 characterizes the case of a partial tracking that Roll (1992) demonstrated for the full tracking. In this way, to calculate the curvature of the TEV frontier in the partial tracking we can use the expression (12) derived from Markowitz's model.

Proposition 2. The TEV frontier generated from a subset of $n$ stocks $(n<N)$ is a shift of the minimum variance frontier obtained from the same subset of stocks.

Proposition 2 presents an interesting difference between full and partial tracking. In full tracking, the TEV frontier is only a shift in the axis of variance of Markowitz's frontier. Therefore, all the tracking portfolios share the same inefficiency $\kappa$, which is identical to the inefficiency of the index that it replicates (Fig. 1). In addition to this shift, a deviation appears in the axis of returns in the partial tracking and this causes the inefficiency in the portfolios in the tracking frontier to vary according to the required

\footnotetext{
${ }^{3}$ The authors will provide the demonstration of the propositions on request.
} 
level of return. In other words, the partial tracking minimum variance frontier and the full tracking frontier are not parallel. This explains why dominance in the mean-variance sense sometimes alternates between the TEV frontier obtained with the partial tracking, and the frontier obtained with the full tracking.

Proposition 3. The TEV frontier curvature generated from a set of $n$ stocks is less than the curvature of the TEV frontier obtained when excluding one or more of those stocks.

Proposition 3 shows that the curvature increases when cardinality of the considered tracking decreases.

\subsection{Criteria weighting in the multiobjective utility function}

The solution of the multiobjective programming model (7) depends on the $w_{i}$ weights set for each of the three parameters considered in the objective function. This section proposes a solution for objectively quantifying these parameters:

Step 1. Apply Algorithm 1 with weights $w_{1}=1$ and $w_{2}=w_{3}=0$. Use the resulting vector $\mathbf{x}_{n}^{*}$ to calculate the weight of the variance criteria of the tracking portfolio: $w_{1}^{*}=1 / V A R_{\mathbf{x}_{n}^{*}}$, being $V A R_{\mathbf{x}_{n}^{*}}$ he variance of the tracking portfolio defined by weight vector $\mathbf{x}_{n}^{*}$.

Step 2. Apply Algorithm 1 with weights $w_{2}=1$ and $w_{1}=w_{3}=0$. Use the resulting vector $\mathbf{x}_{n}^{*}$ to calculate the weights of the TEV criteria: $w_{2}^{*}=1 / T E V_{\mathbf{x}_{n}^{*}}$. Step 3. Apply Algorithm 1 with weights $w_{3}=1$ and $w_{1}=w_{2}=0$. Use the $\mathbf{x}_{n}^{*}$ vector resulting to calculate the weights of the curvature criteria: $w_{3}^{*}=1 / \kappa_{\mathbf{x}_{n}^{*}}$, with $\kappa_{\mathbf{x}_{n}^{*}}$ being the curvature of the TEV frontier generated with the stocks in the tracking portfolio.

The weight of each parameter is fixed in a way that is inversely proportional to the solution - the ideal value - that is obtained when applying Algorithm 1 to the corresponding monoobjective problem. The use of ideal values in the calculation of the $w_{i}$ weights is common in multiobjective programming (Ballestero, Romero 1991) and, more specifically, in compromise programming. However, a trade-off matrix is difficult to obtain, because anti-ideal values can arrive to infinity. For instance, it is easy to calculate the ideal value of the tracking portfolio variance: a positive and limited value. The anti-ideal value is positive but not limited, so the trade-off matrix cannot be calculated.

Even so if the multiobjective frontier does not satisfy the requirements of the investment fund manager, then the weights defined in Steps 1-3 can be changed until a solution is found that better fits the manager's preferences.

\section{Application of the multiobjective model to the partial tracking of the S\&P100 Index}

This section develops an application of the multiobjective model (7) for obtaining tracking frontiers of the S\&P 100. The data set was obtained from the OR-Library (Beasley 1990), which has been used by various researchers for comparing tracking portfolio algorithms (Beasley et al. 2003; Ruiz-Torrubiano, Suárez 2009). The data includes 


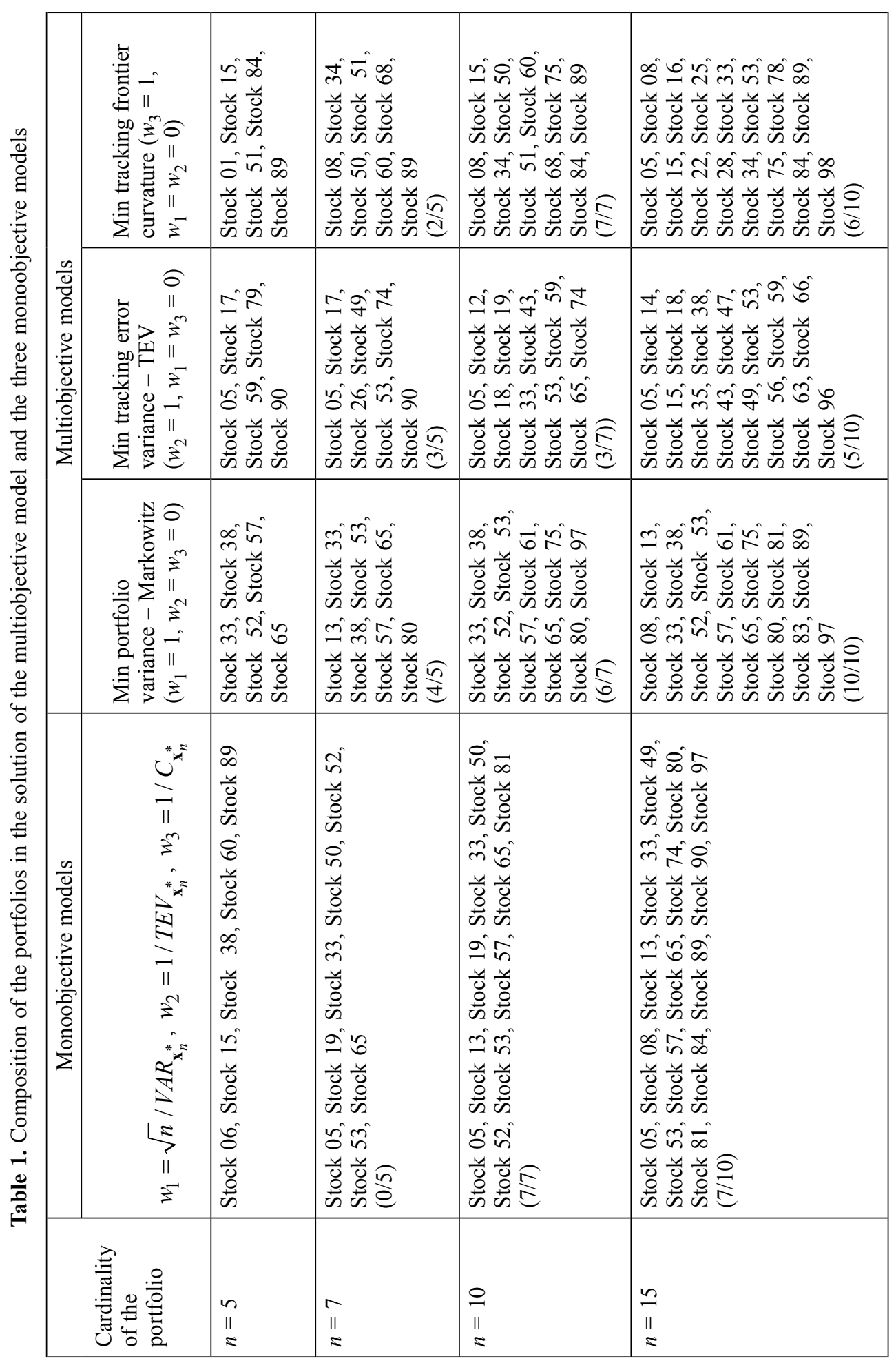


the weekly returns of the index and 98 of its represented stocks during the period 1992-1997. Although the data is not recent, it remains equally valid for illustrating our proposal.

Before obtaining the tracking frontiers, two issues must be resolved prior to the implementation of the multiobjective model: first, the excess return required; and second, the number of stocks in the portfolio. For the first case, the possibility of allowing for negative returns on the underlying index $(G<0)$ was dismissed, as this would assume that the investor is willing to receive a return below the index. We have conservatively assumed that the investor is content with the same return as the index $(G=0)$. With respect to the cardinality of the portfolio, the results are presented considering $5,7,10$, and 15 stocks.

The adapted Tabata and Takeda (1995) (Section 4.1) algorithm was used for the selection of the tracking portfolio stocks. The solution proposed in section 4.3 was used for the $w_{i}$ eights. However, the frontiers obtained were relatively close to the TEV frontier. Accordingly, the criterion of the return variance was over-weighted. Specifically, the weight was multiplied by $\sqrt{n}$. The square root of $n$ was used because it is a function with a negative second derivative.

Table 1 shows the composition of the portfolios for the multiobjective case and the three monoobjective possibilities: minimize the variance of the tracking portfolio - optimization in the sense of Markowitz $\left(w_{1}=1, w_{2}=w_{3}=0\right)$; minimize the TEV $\left(w_{2}=1, w_{1}=\right.$ $\left.w_{3}=0\right)$; and minimize the curvature of the tracking frontier $\left(w_{3}=1, w_{1}=w_{2}=0\right)$. With the minimization of the variance, the portfolio with minimum variance and identical return to the index is obtained $(G=0)$. Using these stocks it is possible to generate a frontier of minimum variance by changing the required return - following Markowitz's classic mean-variance model. With the minimization of TEV the model selects the stocks that also produce the minimum TEV for the case $G=0$, and with these same stocks the corresponding TEV frontier is also generated. Finally, in the model for minimizing the curvature, stocks are selected that minimize this expression and consider excessive returns to be null in the same way. In all cases, the number of stocks in the portfolio was limited to $n$.

Table 1 demonstrates how the composition of the portfolios varies as cardinality increases. Together with the stocks, the ratio between two numbers appears in brackets. The first is the number of stocks that are repeated with respect to the portfolio with immediately inferior cardinality. The second number is the cardinality. For example, for the multiobjective model with $n=15$, there are 7 stocks that are repeated in the multiobjective portfolio with $n=10$. Specifically, these are stocks are $05,13,33,53$, 57, 65 and 81. Therefore, the portfolio with $n=15$ has inherited 7 of the 10 stocks that made up the portfolio with $n=10$ and so the ratio is $7 / 10$. This offers an idea of the persistence with which stocks are held when cardinality increases.

The results show that the mean-variance monoobjective model is the most persistent in its stocks. The portfolio with $n=7$ selects 4 out of 5 stocks from $n=5$; and for $n=$ 10 it is 6 of the 7 possible stocks; while $n=15$ inherits 10 of the possible stocks in the 
$n=10$ portfolio. Of the four models suggested, the model that generates the most variable portfolios is the one that minimizes TEV.

Fig. 2 shows the frontiers obtained for each model on the mean-variance plane according to the cardinalities considered in each case. The frontiers are generated from the stocks shown in Table 1 by simply varying the excess return required. For example, in the case $n=5$, the frontier that minimizes the variance of the portfolio corresponds to Markowitz's classical model when only considering the stocks 33, 38, 52, 57 and 65. These stocks correspond to the minimum variance portfolio for $G=0$ and so the portfolio at this point is less volatile than the other frontiers at the same $G=0$ point.

For nearly the entire spectrum of $G$ values considered in the graph, the minimum variance frontier dominates the two frontiers generated with the monoobjective models: the TEV frontier and the frontier curvature. However, this does not happen with the multiobjective model frontier. For example, in the case $n=5$ it can be seen how the minimum variance frontier dominates the multiobjective frontier for weekly returns of between $0.22 \%$ and $0.37 \%$ (annual returns of $12.1 \%$ and $21.2 \%$ respectively).

The greater curvature of the minimum variance frontier implies that the distance between it and the multiobjective frontier grows rapidly when $|G|$ ncreases. For example, if an investor wants a weekly return of $0.45 \%$, the risk of his position on the minimum variance frontier would be 0.00046 when measured as the variance of return. The investor who chooses the multiobjective frontier would assume a variance of 0.00031 . In other words, the variance recorded at the minimum variance frontier would be $50 \%$ higher than the variance in the multiobjective frontier.

Similar comments can be made for the remaining cardinalities. Fig. 2 shows that as the cardinality of the portfolios increases, the frontier curvature decreases. This means the effect of including the curvature in the multiobjective model is dissipated, because the curvature of the minimum variance frontier is approaching the minimum curvature frontier. The range of returns in which the minimum variance frontier dominates the multiobjective frontier grows, albeit slowly. The difference between the two frontiers also decreases as cardinality increases. Therefore, the S\&P100 can be efficiently tracked with 15 stocks with good results in the mean-variance plane. Adding more stocks to the tracking would not generate an improvement beyond that observed in Fig. 2.

Fig. 3 shows the frontiers in the mean-TEV plane when considering the same cardinalities as in Fig. 2. It can be seen that the frontier that minimizes the TEV approaches the position of the index as cardinality in the portfolio increases. This frontier is preferred in the case of $n=15$ as it dominates the remaining frontiers in all the considered return rates. Something similar occurs with $n=10$. However, if the number of stocks in the portfolio is restricted to 5 , then the excessive curvature means that the multiobjective frontier dominates when returns are below $0.26 \%$ or are greater than $0.37 \%$. This relationship of dominance only becomes clear in the case $n=5$ due to the already mentioned overweighting of the return variance in the multiobjective function (it has been multiplied by $\sqrt{n}$. Similarly, the weight of the criteria can be varied so the multiobjective function tilts towards one in particular, depending on the strategy defined by the fund manager. 

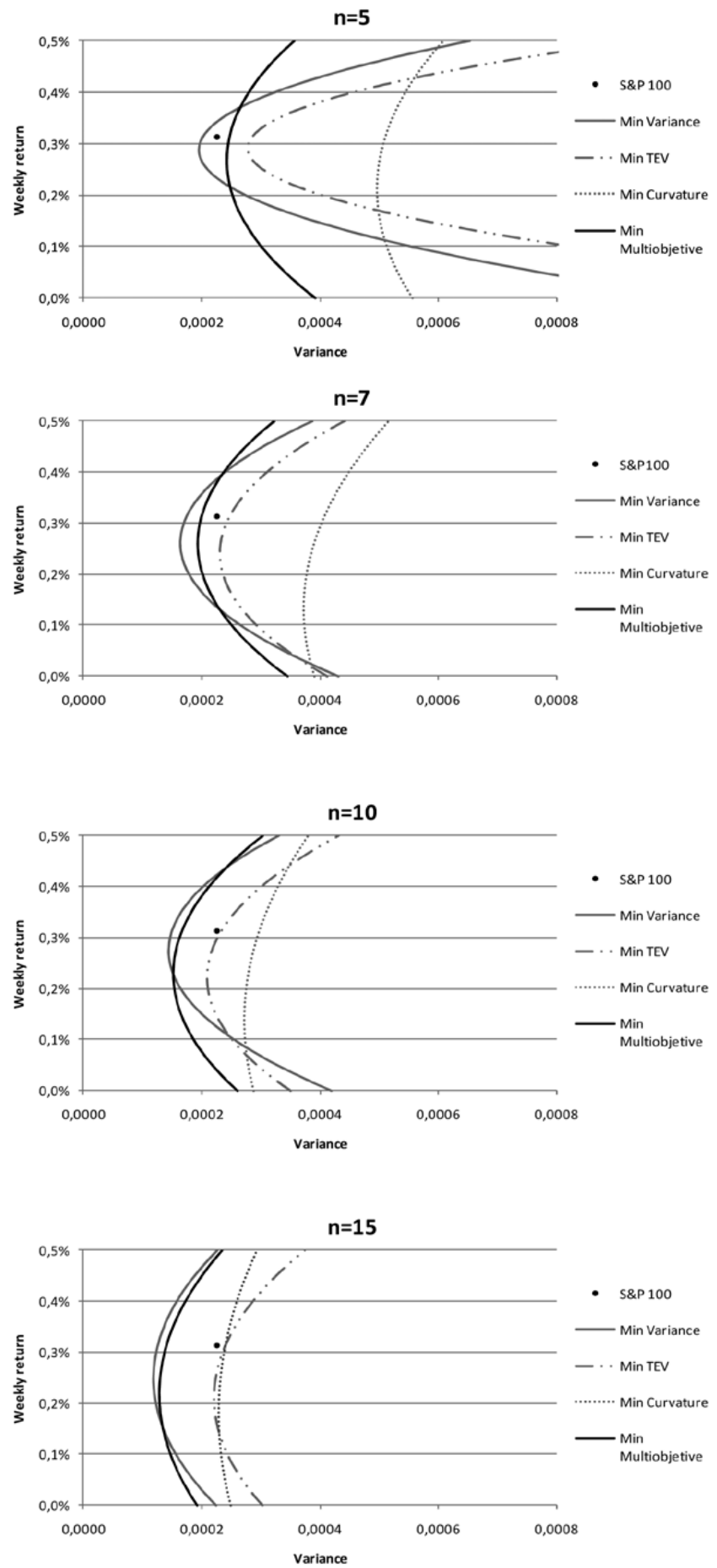

Fig. 2. Graphical representation of the return variance versus the weekly returns for the multiobjective model and the three monoobjective models. Cardinality: $n=5,7,10,15$ 

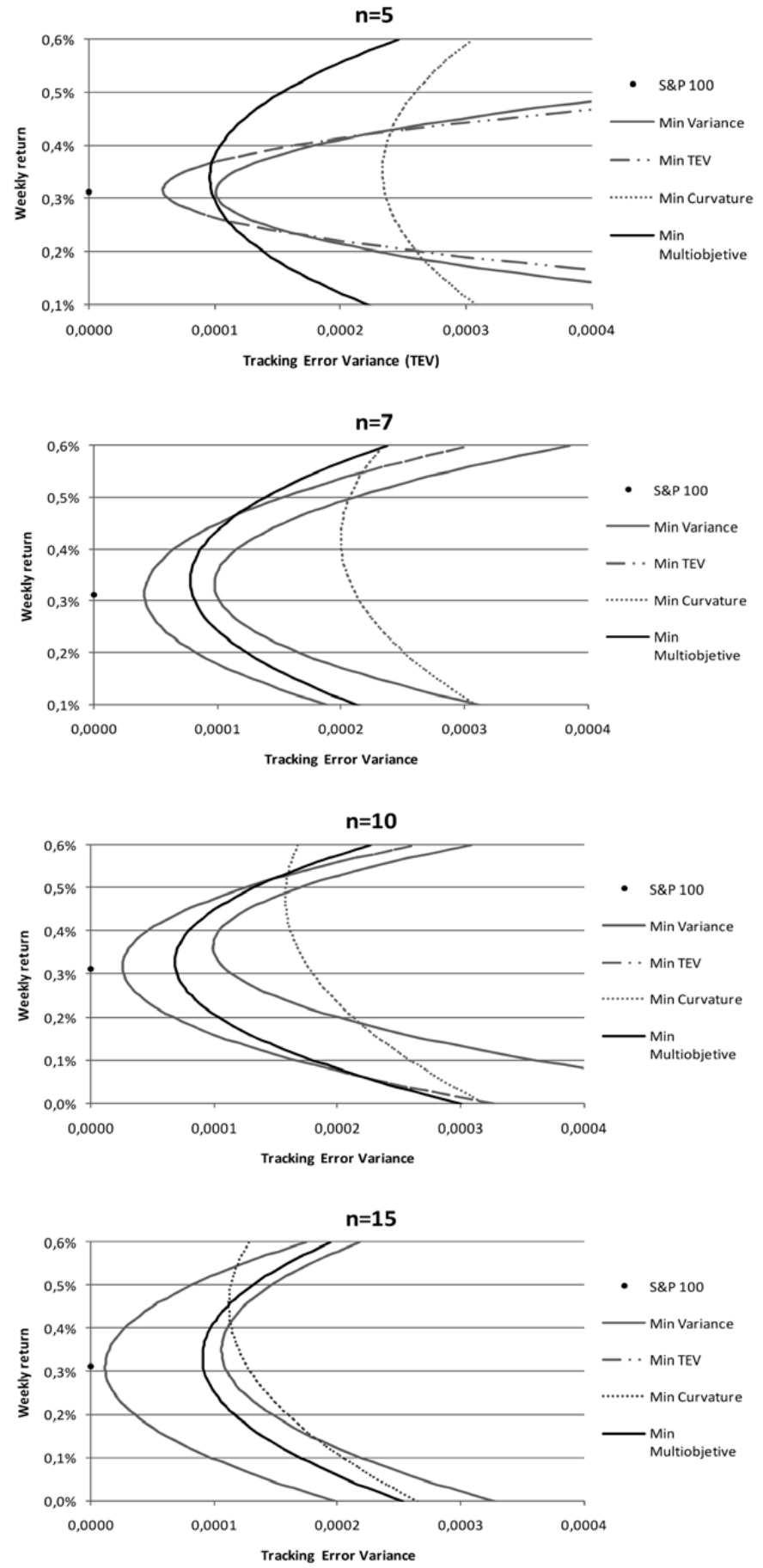

Fig. 3. Graphical representation of the TEV versus the weekly returns for the multiobjective model and the three monoobjective models. Cardinality: $n=5,7,10,15$ 


\section{Conclusions}

Criticisms made about active investment fund management have boosted the success of passive strategies.

Many authors have suggested that costs can be reduced by employing heuristics for the partial tracking of portfolios. Researchers have made use of a limited number of parameters in the selection of these stocks: Tracking error variance (TEV) and return variance. Both criteria are linked to the tracking portfolio, so the portfolio composition varies with the level of return required. Accordingly, different returns can mean that different stocks are considered in the tracking portfolio. In the case a funds' manager wants to cover several risk profiles of his / her clients, he/she should include different stocks in the portfolios, and this represents an increase in transaction costs that reduces the advantages of passive management in comparison to active management.

This paper considers a new parameter for use with the above: Frontier curvature. This criterion is not defined for a given portfolio, but for the set of portfolios that define the tracking frontier. The main implication is that the manager can satisfy different investment profiles using the same subset of stocks, with all the portfolios containing the same stocks and so reducing transaction costs. To appropriately satisfy his / her clients' profiles, only weights in the portfolios shall be properly changed, always working with the same stocks.

For the joint consideration of these criteria we propose the use of multiobjective mathematical programming. In this way the solution can generate a new frontier as a consensus between the frontiers obtained by separately considering each criterion.

The proposed model has been used for tracking the S\&P 100. The results show how the multiobjective frontier is balanced between monoobjective frontiers. From a theoretical viewpoint, the generation of multiobjective solutions is justified for partial tracking portfolios for several reasons.

First, if only the TEV criterion is considered then naive solutions could be obtained in many cases, meaning solutions dominated by stocks with the highest market capitalizations. In such situations, the application of heuristics for building tracking portfolios would not offer a significant advantage with respect to a naive strategy of selecting stocks on the basis of market capitalization.

Second, if only the variance of portfolio returns is considered, then portfolios would be obtained whose future behavior would not necessarily correspond with past behavior. This is one of the main problems with the mean-variance model in which returns and the covariance structure among stocks changes over time - negatively affecting the predictive ability of models. This does not occur with TEV models, where the recent history of stocks satisfactorily explains the evolution of the index. Moreover, these models tend to retain their explanatory power in the future. The reason is simple: there are many stocks that maintain their influence and weight in the composition of the index because of their substantial market capitalizations. 
Third, the inclusion of the curvature of the tracking frontier as a new criterion enables us to contemplate a wider range of investment profiles. With this criterion, it is possible to go beyond the objective of building a single tracking portfolio and to aim for a more general goal: to obtain a tracking frontier that satisfies a larger number of investors by using the same subset of stocks.

\section{References}

Aktan, B.; Korsakiene, R.; Smaliukiene, R. 2010. Time-varying volatility modelling of Baltic stock markets, Journal of Business Economics and Management 11(3): 511-532.

http://dx.doi.org/10.3846/jbem.2010.25

Ballestero, E.; Romero, C. 1991. A theorem connecting utility function optimization and compromise programming, Operations Research Letters 10: 421-427.

http://dx.doi.org/10.1016/0167-6377(91)90045-Q

Beasley, J. E. 1990. OR-Library: distributing test problems by electronic mail, Journal of the Operational Research Society 41(11): 1069-1072.

Beasley, J. E.; Meade, N.; Chang, T. J. 2003. An evolutionary heuristic for the index tracking problem, European Journal of Operational Research 148: 621-643.

http://dx.doi.org/10.1016/S0377-2217(02)00425-3

Canakgoz, N. A.; Beasley, J. E. 2008. Mixed-integer programming approaches for index tracking and enhanced indexation, European Journal of Operational Research 196: 384-399.

http://dx.doi.org/10.1016/j.ejor.2008.03.015

Connor, G.; Leland, H. 1995. Cash management for index tracking, Financial Analysts Journal Nov-Dec: 75-80. http://dx.doi.org/10.2469/faj.v51.n6.1952

Corielli, F.; Marcellino, M. 2006. Factor based index tracking, Journal of Banking \& Finance 30(8): 2215-2233. http://dx.doi.org/10.1016/j.jbankfin.2005.07.012

Derigs, U.; Nickel, N. H. 2004. On a local-search heuristic for a class of tracking error minimization problems in portfolio management, Annals of Operations Research 131: 45-77.

http://dx.doi.org/10.1023/B:ANOR.0000039512.98833.5a

Dose, C.; Cincotti, S. 2005. Clustering of financial time series with application to index and enhanced index tracking portfolio, Physica A 335: 145-151.

http://dx.doi.org/10.1016/j.physa.2005.02.078

Focardi, S. M.; Fabozzi, F. J. 2004. A methodology for index tracking based on time-series clustering, Quantitative Finance 4: 417-425. http://dx.doi.org/10.1080/14697680400008668

Gaivoronoski, A. A.; Krylov, S.; van der Wijst, N. 2005. Optimal portfolio selection and dynamic benchmark tracking, European Journal of Operational Research 163: 115-131.

http://dx.doi.org/10.1016/j.ejor.2003.12.001

Hallerbach, W. G.; Spronk, J. 2002. The relevance of MCDM for financial decisions, Journal of Multi-Criteria Decision Analysis 11(4-5): 187-195. http://dx.doi.org/10.1002/mcda.328

Jarrett, J. E.; Schilling, J. 2008. Daily variation and predicting stock market returns for the Frankfürter Börse (stock market), Journal of Business Economics and Management 9(3): 189-198. http://dx.doi.org/10.3846/1611-1699.2008.9.189-198

Markowitz, H. M. 1952. Portfolio selection, Journal of Finance 7(1): 77-91.

Markowitz, H. M. 1959. Portfolio selection: efficient diversification of investments. New York: Wiley.

Roll. R. 1992. A mean/variance analysis of tracking error, The Journal of Portfolio Management 18: $13-22$. 
Rudolf, M.; Wolter, H.-J.; Zimmermann, H. 1999. A linear model for tracking error minimization, Journal of Banking \& Finance 23(1): 85-103. http://dx.doi.org/10.1016/S0378-4266(98)00076-4 Ruiz-Torrubiano, R.; Suárez, A. 2009. A hybrid optimization approach to index tracking, Annals of Operations Research 166: 57-71. http://dx.doi.org/10.1007/s10479-008-0404-4

Rutkauskas, A.V.; Stasytyte, V. 2007. Decision making strategies in global exchange and capital markets, in K. Elleithy (Ed.). Advances and innovations in systems, computing sciences and software engineering. Springer, 17-22.

Steuer, R. E. 1986. Multiple criteria optimization. Theory computation and application. New York: Wiley.

Tabata, Y.; Takeda, E. 1995. Bicriteria optimization problem of designing an index fund, Journal of the Operational Research Society 46(8): 1023-1032.

Teresiene, D. 2009. Lithuanian stock market analysis using a set of GARCH models, Journal of Business Economics and Management 10(4): 349-360.

http://dx.doi.org/10.3846/1611-1699.2009.10.349-360

Wu, L. C.; Chou, S. C.; Yang, C. C.; Ong, C. S 2007. Enhanced index investing based on goal programming, The Journal of Portfolio Management Spring: 49-56.

http://dx.doi.org/10.3905/jpm.2007.684753

Zeleny, M. 1982. Multiple criteria decision making. New York: McGraw-Hill.

Fernando GARCIA. PhD, is Associate Professor of Finance at the Faculty of Business Administration and Management of the Universidad Politécnica de Valencia. He has published several refereed papers in international journals and international congress proceedings. His research interest focuses on firm performance, globalization, risk analysis and investment.

Francisco GUIJARRO. PhD, is Lecturer of Finance at the Faculty of Business Administration and Management of the Universidad Politécnica de Valencia. He has published several refereed papers in the European Journal of Operational Research, Journal of the Operational Research Society, Annals of Operations Research, Computers \& Operations Research, etc.

Ismael MOYA. PhD, is Professor of Finance and Dean at the Faculty of Business Administration and Management of the Universidad Politécnica de Valencia. He has published several refereed papers in Annals of Public and Cooperative Economics, Energy Economics, European Journal of Operational Research, Computers \& Operations Research, Information World, etc. 